\title{
Radionuclide left ventricular contractile indices and their relationship to heart size in dogs
}

\begin{abstract}
To identify possible mechanisms to explain differences between the maximum time-varying elastance $\left(E_{\max }\right)$ and end-systolic pressure-volume $\left(E_{e s}\right)$ slope values calculated with radionuclide anglography and to establlsh whether they have a relationship to heart size, we studied 16 dogs that were instrumented with micromanometer left ventricular catheters and had red blood cells tagged with technetium-99m for radionuclide angiography. Hemodynamics and radionuclide angiograms were obtained under control conditions and during six additional steady-state loading conditlons. Isochronal $E_{\max }$ averaged $7.14 \pm 2.54 \mathrm{~mm} \mathrm{Hg} / \mathrm{ml}$, while $E_{e s}$ averaged $5.68 \pm 1.88 \mathrm{~mm}$ $\mathrm{Hg} / \mathrm{ml}(\mathrm{p}<0.01)$, but they were highly correlated $(r=0.95, p<0.001)$. This observation was related to the assumption of linearity when curvilinearity was present and to the important influence of timing on these relationships. The $E_{\max }$ and $E_{e s}$ slope values were compared to dog welght; left ventricular weight, which ranged from 85 to $142 \mathrm{gm}$ (mean $113 \pm 18 \mathrm{gm}$ ); and left ventricular end-diastolic volume, which ranged from 15 to $56 \mathrm{ml}$ (mean $29 \pm 10 \mathrm{ml}$ ) using multiple regression analyses. The $E_{\max }$ and $E_{e s}$ slope values demonstrated a comparable inverse linear relationship with only left ventricular end-diastolic volume $(r=0.76$ and $-0.69, p<0.001$ and $p<0.01$ ). We conclude that the differences between $E_{\max }$ and $E_{e s}$ slope values calculated with radionuclide anglography are related to the assumption of linearity when curvilinearity is present and to the importance of the timing of systolic events and that both $E_{\max }$ and $E_{a s}$ are comparably related to left ventricular end-diastolic volume. Since the $E_{\max }$ and $E_{\text {es }}$ slope values are affected by similar influences, are highly correlated, and are comparably related to heart size, they both may be useful for assessing alterations in left ventricular contractlity as long as they are applied consistently in animals or man. (AM HEART J 1989;118:325.)
\end{abstract}

Mark R. Starling, MD, G. B. John Mancini, MD, Daniel G. Montgomery, BS, and Milton D. Gross, MD. Ann Arbor, Mich.

Left ventricular contractility has been assessed in the excised, supported left ventricle, ${ }^{1-3}$ in various intact animal preparations, ${ }^{4}$ and in $\operatorname{man}^{5-8}$ by means of the time-varying elastance concept. The maximum elastance $\left(E_{\max }\right)$ calculated using isochronal pressure-volume data points from biplane contrast cineangiography ${ }^{6}$ or radionuclide angiography ${ }^{8}$ has been shown to differ, on the average, from other indices of left ventricular contractility calculated at more commonly defined measures of end-ejection in

From the Department of Internal Medicine, Divisions of Cardiology and Nuclear Medicine, University of Michigan and Veterans Administration Medical Centers.

Supported by Grant NIH R01-HL36450 from the National Heart, Lung, and Blood Institute of the National Institutes of Health, Bethesda, Maryland; by the American Heart Association, Michigan Affiliate, Iathrup Village Mich.; and by the Veterans Administration, Washington, D.C. Dr. Starling is the recipient of NIH Research Career Development Award K04-HL01787 from the National Heart, Lung, and Blood Institute, Bethesda, Md.

Received for publication Feb. 2, 1989; accepted March 20, 1989.

Reprint requests: Mark R. Starling, MD, University of Michigan, Dept. of Internal Medicine, Division of Cardiology, VA Medical Center, 2215 Fuller Rd., Ann Arbor, MI 48105. man. Although several possible explanations exist, no specific mechanisms have been identified to explain the differences between $\mathrm{E}_{\max }$ and other contractile indices, specifically end-systolic pressure-volume relations $\left(\mathrm{E}_{\mathrm{eg}}\right)$.

The $\mathrm{E}_{\mathrm{es}}$ slope values have been shown to be related to heart size in animals. ${ }^{9}, 10$ The $\mathrm{E}_{\max }$ and $\mathrm{E}_{\mathrm{es}}$ slope values calculated with biplane contrast cineangiography in patients with normal left ventricular size and performance have also demonstrated an inverse linear relationship with left ventricular mass and end-diastolic volume. ${ }^{6}$ The $\mathrm{E}_{\max }$ slope values calculated with radionuclide angiography are comparable to those obtained with biplane cineangiography in man, ${ }^{7}$ but whether the $\mathrm{E}_{\max }$ and $\mathrm{E}_{\mathrm{es}}$ slope values calculated with radionuclide angiography are also related to heart size can only be presumed. Accordingly, the purposes of this investigation were: (1) to elucidate the possible mechanisms for differences in $E_{\max }$ and $E_{e s}$ slope values calculated with radionuclide angiography, if such differences exist, and (2) to determine whether a relationship with heart size ex- 
Table I. Contractile index and heart size data $(n=16)$

\begin{tabular}{|c|c|c|c|c|c|c|c|}
\hline $\begin{array}{l}\text { Dog } \\
\text { No. }\end{array}$ & $\begin{array}{c}E_{\max } \\
(m m \mathrm{Hg} / \mathrm{ml})\end{array}$ & $\begin{array}{c}E_{\max } V_{0} \\
(m l)\end{array}$ & $\begin{array}{c}E_{e s} \\
(m m H g / m l)\end{array}$ & $\begin{array}{c}E_{e s} V_{o} \\
(m l)\end{array}$ & $\begin{array}{c}\text { Dog } w t \\
(\mathrm{~kg})\end{array}$ & $\begin{array}{c}L V w t \\
(g m)\end{array}$ & $\begin{array}{c}E D V \\
(m l)\end{array}$ \\
\hline 1 & 5.87 & 1.8 & 5.65 & 0.60 & 27.10 & 113.4 & 27 \\
\hline 2 & 10.36 & 1.7 & 8.04 & -1.80 & 25.00 & 96.0 & 16 \\
\hline 3 & 6.97 & 19.7 & 5.12 & 10.40 & 31.50 & 141.5 & 33 \\
\hline 4 & 8.39 & 5.9 & 6.49 & 2.30 & 18.70 & 84.7 & 28 \\
\hline 5 & 2.76 & 10.7 & 2.52 & 5.20 & 29.50 & 121.9 & 56 \\
\hline 6 & 6.16 & 22.8 & 3.96 & 4.10 & 38.60 & 134.3 & 31 \\
\hline 7 & 6.45 & 16.4 & 4.80 & 5.70 & 23.90 & 130.1 & 30 \\
\hline 8 & 5.03 & 3.6 & 4.72 & 1.90 & 22.70 & 136.3 & 31 \\
\hline 9 & 6.88 & 2.3 & 5.74 & -3.00 & 22.70 & 113.9 & 21 \\
\hline 10 & 5.39 & 5.4 & 3.69 & -9.30 & 27.50 & 122.0 & 26 \\
\hline 11 & 12.10 & 5.2 & 9.12 & -0.50 & 20.60 & 94.8 & 15 \\
\hline 12 & 11.61 & 3.3 & 8.88 & -1.70 & 19.50 & 90.7 & 22 \\
\hline 13 & 4.18 & -10.0 & 4.05 & -11.80 & 25.60 & 109.9 & 42 \\
\hline 14 & 7.24 & 18.7 & 6.92 & 17.20 & 24.30 & 126.3 & 37 \\
\hline 15 & 8.35 & 5.0 & 6.47 & 0.50 & 22.20 & 98.5 & 30 \\
\hline 16 & 6.50 & 4.6 & 4.75 & -4.50 & 18.90 & 91.5 & 23 \\
\hline Mean \pm S.D & $7.14 \pm 2.54$ & $7 \pm 8$ & $5.68 \pm 1.88$ & $1 \pm 7$ & $24.89 \pm 5.20$ & $112.9 \pm 18.3$ & $29 \pm 10$ \\
\hline
\end{tabular}

$E_{\max }$, Maximum time-varying elastance; $\mathrm{V}_{0}$, unstressed volume; $\mathrm{E}_{\mathrm{es}}$, end-systolic pressure-volume; wt, weight; $E D V$, end-diastolic volume; $L V$, left ventricle.

ists for either $\mathrm{E}_{\max }$ or $\mathrm{E}_{\mathrm{es}}$ slope values calculated with radionuclide angiography in dogs.

\section{METHODS}

Animal instrumentation. Sixteen dogs of either sex, weighing 20 to $39 \mathrm{~kg}$, were anesthetized with intravenous pentobarbital sodium $(35 \mathrm{mg} / \mathrm{kg})$, intubated, and ventilated with $15 \mathrm{ml} / \mathrm{kg}$ oxygen-enriched room air. A left carotid arteriotomy and internal jugular venotomy were performed for vascular access. Electrocardiographic limb leads were attached to monitor heart rate. Through the fifth left intercostal space, a thoracotomy was performed, and the descending thoracic aorta and inferior vena cava were encircled with elastic snares. The pericardium was then incised parallel to the phrenic nerve and was left unopposed. A precalibrated micromanometer catheter (Millar Instruments, Houston, Texas) was inserted into the left ventricle through an apical stab wound and was secured. Finally, each animal was anticoagulated with 3000 units of heparin.

Protocol. Each animal was positioned under the gamma scintillation camera in an obliquity that optimally separated the left ventricle from the right ventricle in the plane of the interventricular septum. During each radionuclide acquisition, an electrocardiogram, micromanometer left ventricular pressures, and the first derivative of left ventricular pressure $(\mathrm{dP} / \mathrm{dt})$ were recorded on a Gould $2800 \mathrm{~S}$ eight-channel physiologic recorder (Gould Electronics, Cleveland, Ohio). All data were acquired under control conditions and during a maximum of six additional, steadystate hemodynamic conditions produced by either aortic or inferior vena caval occlusion. At the completion of the protocol, each animal was killed. The distance from the geometric center of the left ventricle to the chest wall (camera crystal) was then measured. At that time the hearts were excised, structures were dissected free from the left ventricle, and the left ventricles were weighed (in grams).
Hemodynamics. The micromanometer left ventricular pressure signals were averaged, and the average leît ventricular pressure waveforms were hand digitized at $200 \mathrm{~Hz}$ using a Calcomp 9100 interfaced to an IBM PC computer (IBM Corp., Purchase, N.Y.). The program developed in our laboratory provides instantaneous left ventricular pressure and the first derivative of left ventricular pressure, $\mathrm{dP} / \mathrm{dt}^{6-8,11}$ These hand-digitized left ventricular pressure data were interpolated to guarantee the isochronicity of the pressure-volume data points.

Radionuclide anglography. Red blood cells were tagged by means of a modified in vitro labeling technique with 30 $\mathrm{mCi}$ of technetium-99m. A standard field of view gamma scintillation camera (Siemens Gammasonics, Inc. Nuclear Medicine Division, Des Plaines, Ill.) was used to acquire radionuclide images under control conditions and during each hemodynamic steady-state condition into $64 \times 64$ byte matrices for consecutive, corresponding $20 \mathrm{msec}$ frames until information from $500 \mathrm{R}-\mathrm{R}$ intervals was processed. Midway through the radionuclide acquisition, a 2 $\mathrm{ml}$ blood sample was obtained and the time was recorded. Each blood sample was counted at the completion of the protocol on the collimator for 2 minutes to obtain counts $/ 2$ $\mathrm{min} / 2 \mathrm{ml}$. The distance from the gamma scintillation camera to the left ventricular geometric center of mass was ob tained at the completion of the protocol.

Radionuclide left ventricular volumes were calculated by means of a hand-drawn region-of-interest technique to obtain left ventricular count data. ${ }^{12,13}$ Briefly, the left ventricular images were background-subtracted and smoothed. Left ventricular regions-of-interest were outlined by the operator for each frame. Then these background-subtracted left ventricular counts were standardized for frame duration, cardiac cycles acquired, and decay-corrected blood sample counts. These standardized left ventricular counts were corrected for photon attenuation $\left(e^{\mathrm{ud}}\right)$, where $u$ is assumed to represent the linear attenuation coefficient 
of $0.15 \mathrm{~cm}^{-1}$ for the $140 \mathrm{keV}$ photon of technetium $-99 \mathrm{~m}$ and $d$ represents the directly measured distance to the left ventricular geometric center of mass.

Calculation of left ventricular $E_{\max }$ and $E_{\text {es }}$ slope values. The corresponding micromanometer left ventricular pressures and radionuclide left ventricular volumes for each loading condition were plotted to obtain multiple pressure-volume loops for each animal. Then isochronal pressure-volume data points from each of the pressurevolume loops were subjected to linear regression analysis to obtain the maximum slope of these relations, which was defined as $\mathrm{E}_{\max }{ }^{1-8}$ The $\mathrm{E}_{\text {es }}$ relations were calculated by determining the maximal pressure-volume ratio, and then subjecting these pressure-volume data points to linear regression analysis to obtain a slope.6, 8, 14-19

Statistical analysis. All data are presented as the mean \pm standard deviation. The $E_{\max }$ slope values were compared to the $\mathrm{E}_{\mathrm{es}}$ slope values by a paired $t$ test. The unstressed volumes $\left(\mathrm{V}_{0}\right)$ for these indices of left ventricular contractility were also compared by a paired $t$ test. The $\mathrm{E}_{\max }$ and $\mathrm{E}_{\text {es }}$ slope and $\mathrm{V}_{0}$ values were also compared by linear regression analysis. A $z$ test for parallelism was performed to establish whether the $E_{\max }$ and $E_{e s}$ slope values differed in an individual dog. The $\mathrm{E}_{\max }$ and $\mathrm{E}_{\mathrm{es}}$ slope values were compared to dog weight, left ventricular weight, and left ventricular end-diastolic volume (defined as the maximum left ventricular volume obtained from the radionuclide time-activity curve) using multiple regression analysis (MIDAS, University of Michigan, 1976). A probability value of 0.05 or less was required for significance.

\section{RESULTS}

Indices of left ventricular contractility. The individual and mean $\mathrm{E}_{\max }$ and $\mathrm{E}_{\mathrm{es}}$ slope values are shown in Table 1 and Fig. 1 . The $E_{\max }$ slope values ranged from 2.76 to $12.10 \mathrm{~mm} \mathrm{Hg} / \mathrm{ml}$, with a mean of $7.14 \pm 2.54$ $\mathrm{mm} \mathrm{Hg} / \mathrm{ml}$. The $E_{e s}$ slope values ranged from 2.52 to $9.12 \mathrm{~mm} \mathrm{Hg} / \mathrm{ml}$, with a mean of $5.68 \pm 1.88 \mathrm{~mm} \mathrm{Hg} /$ $\mathrm{ml}$. The average $E_{\max }$ slope value exceeded that for $\mathrm{E}_{\mathrm{es}}(p<0.01$, Fig. 1, left panel). In spite of a significant difference in the mean $\mathrm{E}_{\max }$ and $\mathrm{E}_{\mathrm{es}}$ slope values, a separate issue is whether the $\mathrm{E}_{\max }$ and $\mathrm{E}_{\mathrm{es}}$ slope values calculated using the present methodology differ in an individual dog. The individual $E_{\max }$ and $\mathrm{E}_{\mathrm{es}}$ slope values did not differ significantly when a $z$ test for parallelism was used to compare them in each $\operatorname{dog}(p>0.05$ for all comparisons). This lack of a significant difference between the individual $\mathrm{E}_{\max }$ and $E_{\text {es }}$ slope values occurred in spite of the generation of as many as seven pressure-volume loops. Moreover, the individual $\mathrm{E}_{\mathrm{es}}$ slope values correlated with their corresponding $\mathrm{E}_{\max }$ slope values $(r=0.95$ and $p<$ 0.001 , Fig. 2, left panel).

The extrapolated volume-axis intercepts for the $\mathrm{E}_{\max }$ slope values ranged from -10 to $23 \mathrm{ml}$, with an average $V_{0}$ of $7 \pm 8 \mathrm{ml}$. The $E_{e s}$ slope values had unstressed volumes that ranged from -12 to 17 , with a mean of $1 \pm 7 \mathrm{ml}$. These $V_{0}$ values differed signifi-

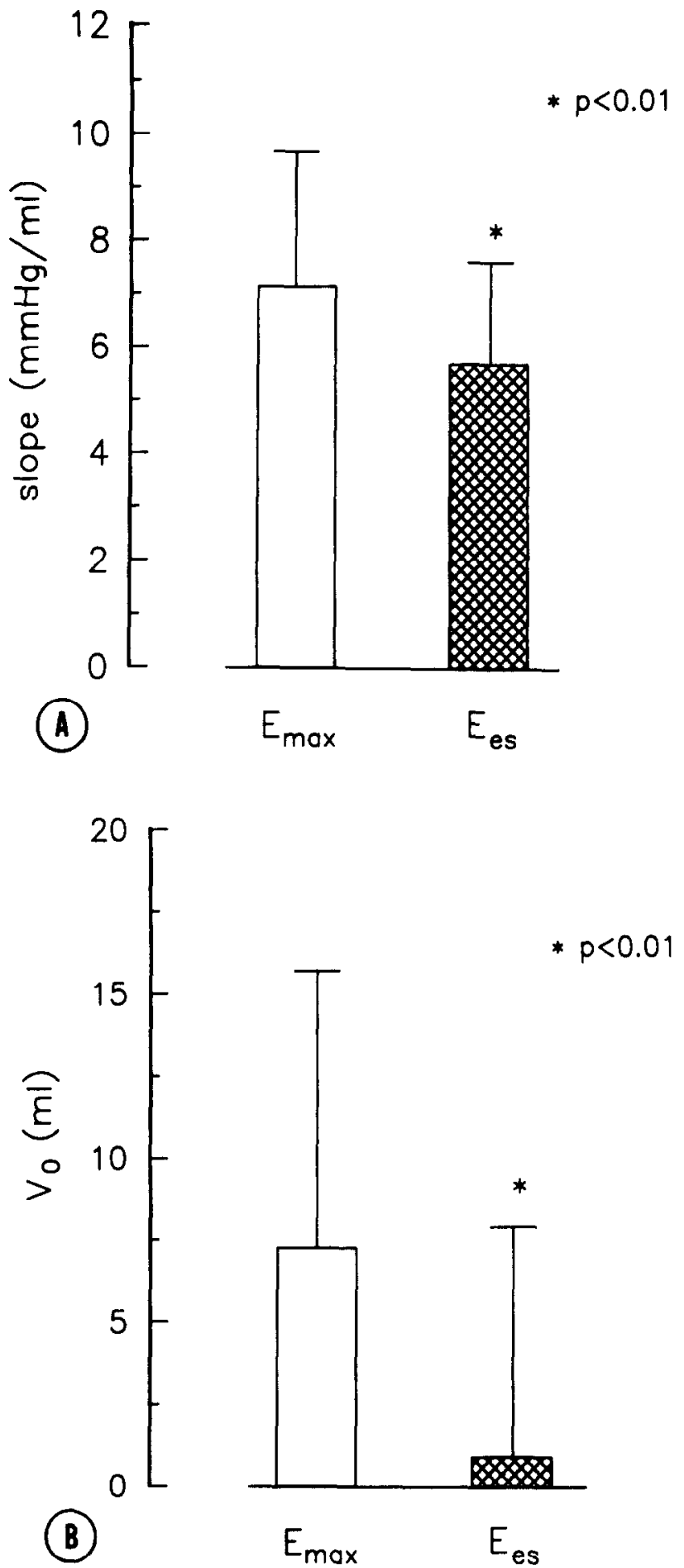

Fig. 1. A, The mean $\mathrm{E}_{\max }$ (open bar) and the mean $\mathrm{E}_{\mathrm{es}}$ (cross-hatched bar) slope values from the 16 animals are shown. A significant difference is noted. $\mathbf{B}$, The mean $\mathbf{E}_{\max }$ (open bar) and the mean $\mathrm{E}_{\mathrm{es}}$ (cross-hatched bar) unstressed volumes $\left(V_{0}\right)$ are shown. A significant difference is noted.

cantly ( $p<0.01$, Fig. 1, right panel). The $\mathrm{V}_{\mathrm{O}}$ s for the $\mathrm{E}_{\mathrm{es}}$ relations correlated, however, with those for $\mathrm{E}_{\max }$ $(r=0.80, p<0.001$, Fig. 2, right panel $)$.

To further elucidate the possible mechanisms for 

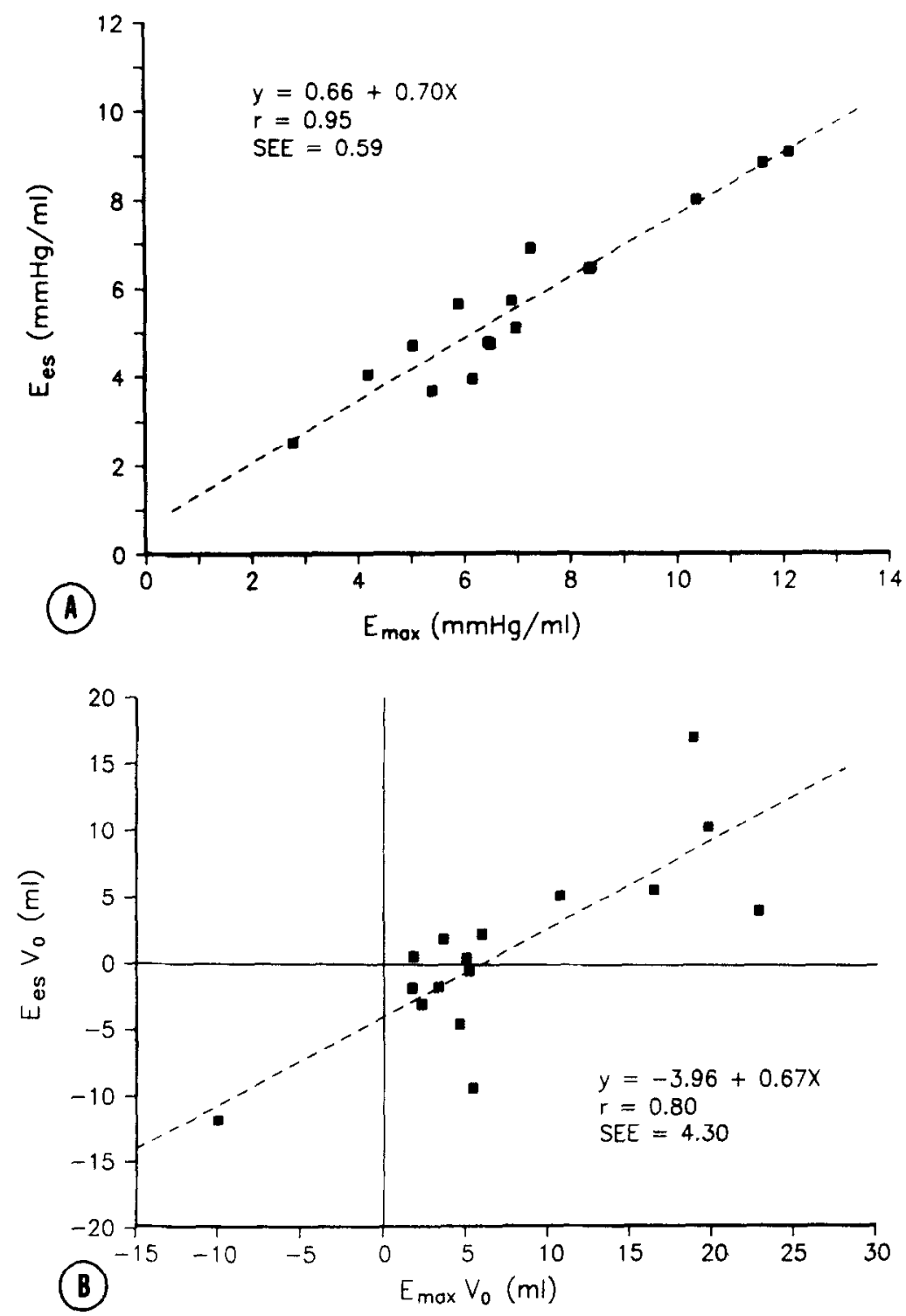

Fig. 2. $A$, The individual $\mathrm{E}_{\mathrm{es}}$ slope values (on the ordinate) are compared to the individual $\mathrm{E}_{\max }$ slope values (on the abscissa). The individual data points, regression line, regression equation, correlation coefficient $(r)$. and standard error of the estimate ( $S E E$ ) are shown. $\mathrm{B}$, The individual $\mathrm{E}_{\mathrm{es}}$ unstressed volumes (on the ordinate) are compared to the individual $\mathrm{E}_{\max }$ unstressed volumes (on the abscissa) in a similar fashion.

these observations, we investigated the individual $E_{\max }$ and $E_{e s}$ curves for each dog. The $E_{\max }$ and $E_{e s}$ slope values were essentially identical in 5 of the 16 dogs, while they differed by more than $1 \mathrm{~mm} \mathrm{Hg} / \mathrm{ml}$ in the remaining 11 dogs. Two possible explanations emerged. First, as shown in Fig. 3, in the higher contractile range ( $>6 \mathrm{~mm} \mathrm{Hg} / \mathrm{ml})$, there was curvilinearity to these relationships. When a polynomial curve fit to these data was performed, the $E_{\max }$ and $E_{\text {es }}$ relations became nearly superimposable with comparable $\mathrm{V}_{\mathrm{O}}$. Second, also shown in Fig. 3, the timevarying elastance function appeared to go beyond the maximum pressure-volume point in some dogs on those pressure-volume loops generated following in- ferior vena caval occlusion, but not generally on those pressure-volume loops generated under control conditions or following aortic occlusion. The timevarying elastance did not, however, go beyond minimum ventricular volume, and it did not exceed the maximum pressure-volume point by more than 20 msec in this preparation.

Comparisons with heart size. When the $\mathrm{E}_{\max }$ slope values were compared to dog weight, left ventricular weight, and left ventricular end-diastolic volume, they demonstrated an inverse linear relationship with left ventricular mass and end-diastolic volume $(r=-0.59$ and $-0.76, p<0.05$ and $<0.001)$. When the $\mathrm{E}_{\mathrm{es}}$ slope values were compared with these mea- 


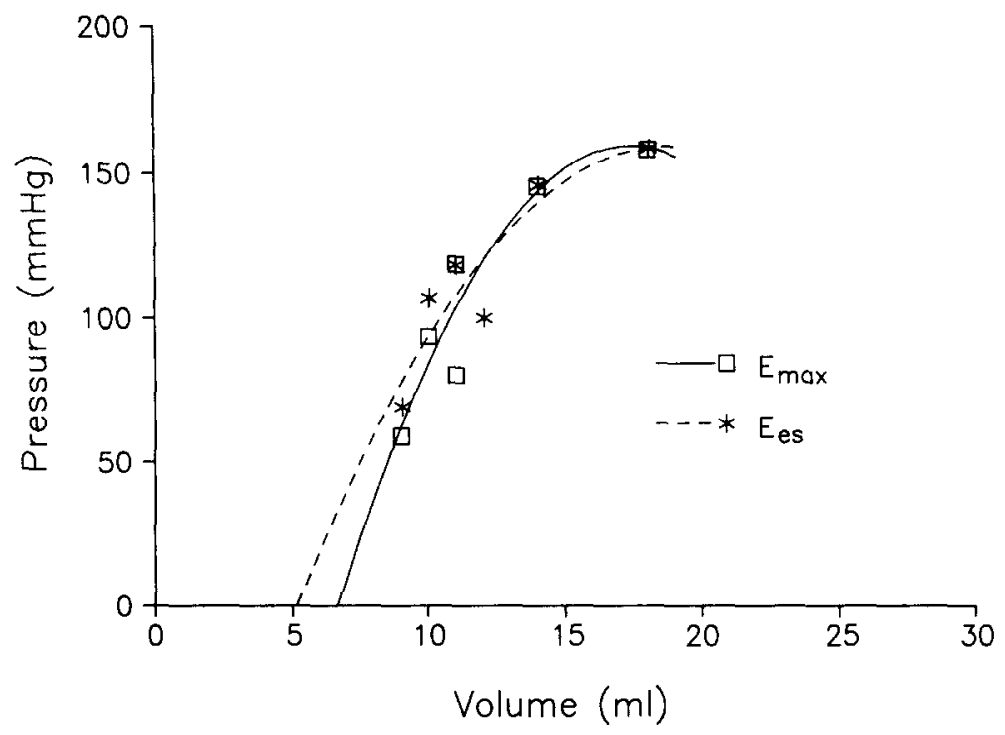

Fig. 3. Curvilinearity to the $\mathrm{E}_{\max }$ and $\mathrm{E}_{\mathrm{es}}$ relations in the high contractile range are shown. Note that when a polynomial curve fit to the individual $\mathrm{E}_{\max }$ and $\mathrm{E}_{\mathrm{es}}$ pressure-volume data points is applied, there is no difference in the two relations.

sures, they demonstrated an inverse linear relationship with dog weight $(r=-0.54, p<0.05)$, left ventricular weight $(r=-0.59, p<0.05)$, and left ventricular end-diastolic volume $(r=-0.69, p<0.01)$. When multiple regression analyses were applied to these data, the $E_{\max }$ and $E_{\text {es }}$ slope values demonstrated relationships only with left ventricular enddiastolic volume (Fig. 4).

When the $\mathrm{E}_{\max } \mathrm{V}_{\mathrm{O}}$ s were compared to dog weight, left ventricular weight, and left ventricular end-diastolic volume, the $\mathrm{V}_{\mathrm{O}}$ values demonstrated a relationship with dog weight $(r=0.53, p<.05)$ and left ventricular weight $(r=0.57, p=0.02)$, but not with left ventricular end-diastolic volume. When the $\mathrm{E}_{\mathrm{es}} \mathrm{V}_{\mathrm{O}} \mathrm{s}$ were compared with these measures, no consistent relationship was evident.

\section{DISCUSSION}

The data from the present investigation, in which radionuclide angiography was used to calculate left ventricular contractile indices, indicate that the average $E_{\max }$ and $E_{\text {es }}$ slope values differ. The apparent mechanisms for this observation are the assumption of linearity when curvilinearity is present and the dependency of these slopes on the timing of systolic events in some dogs. An inverse linear relationship was demonstrated between the individual $\mathrm{E}_{\max }$ and $E_{e s}$ slope values and their corresponding left ventricular end-diastolic volumes. Only the $\mathrm{E}_{\max }$ slope values had $V_{0}$ that demonstrated a relationship with heart size, while the $E_{\mathrm{es}} \mathrm{V}_{\mathrm{O}}$ s had no relationship to animal or heart size. Thus the average $\mathrm{E}_{\max }$ and $\mathrm{E}_{\mathrm{es}}$ slope values differ when the potential for curvilin- earity and the timing of systolic events is ignored, and these contractile indices both have a comparable relationship with heart size that is best represented by left ventricular end-diastolic volume.

It has previously been reported that $\mathrm{E}_{\max }$ slope values calculated with biplane contrast cineangiography ${ }^{6}$ and radionuclide angiography $5,7,8$ in man are underestimated by other indices of left ventricular contractility. The explanation for these observations has not been completely elucidated, but several possibilities exist. First, in the animals with higher slope values, curvilinearity affected the assumptions regarding linearity of and the observations regarding differences between the $\mathrm{E}_{\max }$ and $\mathrm{E}_{\mathrm{es}}$ slope values in the present investigation. This is consistent with the observations of Burkhoff et al. ${ }^{20}$ in an excised supported left ventricle, and of Little et al. ${ }^{21}$ in chronically instrumented animals. Little et al. ${ }^{21}$ reported that, despite significant linear fits to the $\mathbf{E}_{\mathrm{es}}$ data, curvilinearity shifted the relationship to a higher $\mathrm{V}_{0}$, which was noted in this investigation for $\mathrm{E}_{\mathrm{es}}$ more so than for $E_{\max }$. Compensation for curvilinearity caused the $\mathrm{E}_{\mathrm{es}}$ relations to approximate more closely the $E_{\max }$ relations in some dogs. Second, there may also be an important timing phenomenon in some patients or animals 6 - that is, the $\mathrm{E}_{\max }$ slope value may include pressure-volume data points beyond the maximum pressure-volume point but not beyond minimum ventricular volume in the unloaded pressure-volume loops. This observation contrasts with what Kass et al..$^{22}$ have suggested and with what has been shown for indices of regional left ventricular contractility by Miller et al. ${ }^{23}$ They reported that the 

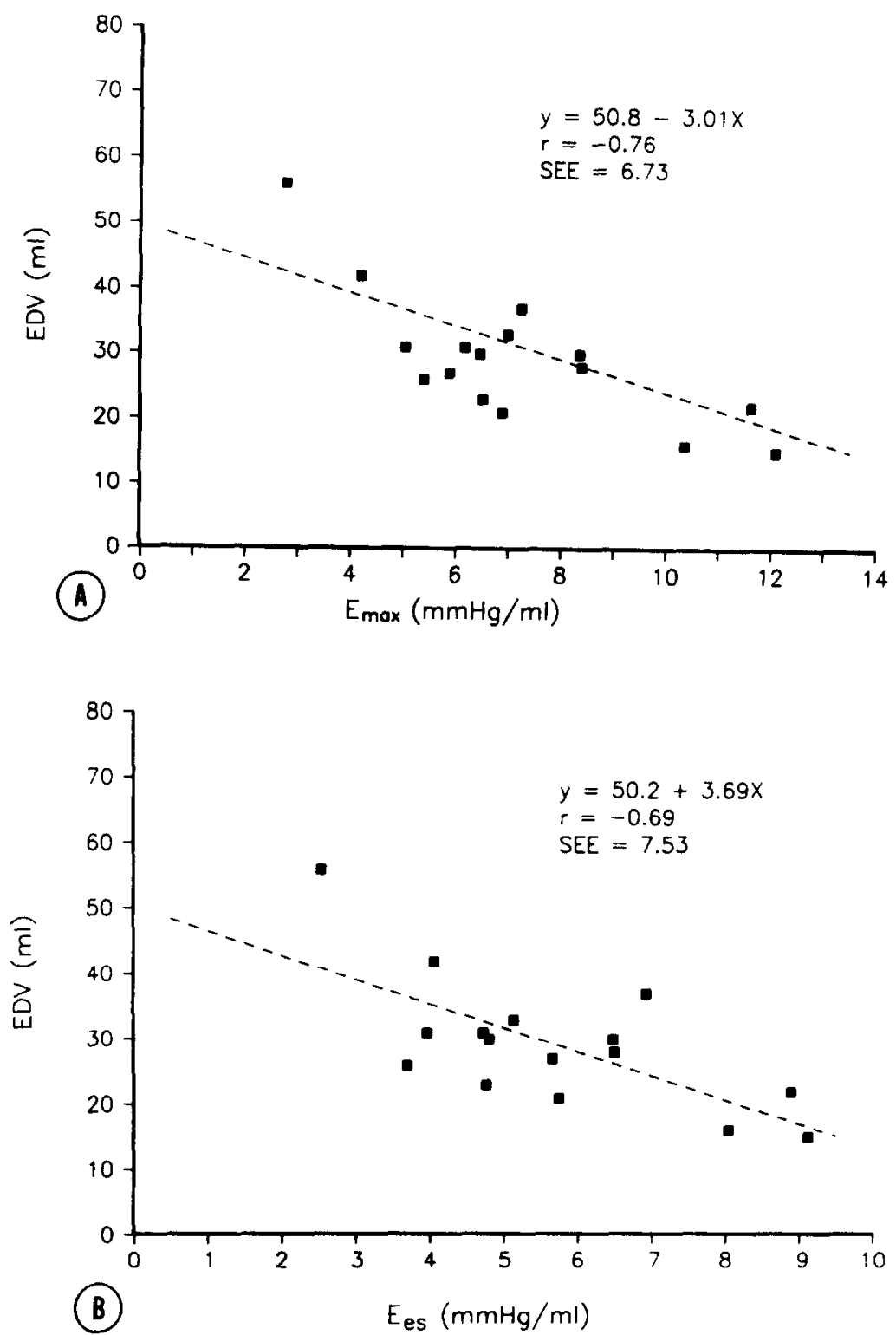

Fig. 4. $\mathrm{A}$, The individual end-diastolic volumes $(E D V)$ (on the ordinate) are compared to the individual $\mathrm{E}_{\max }$ slope values (on the abscissa). The individual data points, regression line, regression equation, correlation coefficient $(r)$, and standard error of the estimate (SEE) are shown. B, The left ventricular end-diastolic volumes (on the ordinate) are compared to the $\mathrm{E}_{\mathrm{es}}$ slope values (on the abscissa) in a similar fashion.

$\mathrm{E}_{\max }$ slope values may be constructed from pressurevolume points ${ }^{22}$ or pressure-segment length (wall thickness) points, ${ }^{23}$ which all reside well beyond the maximal pressure-volume point of each loop. We did not make this observation in our study. Nevertheless, the present observations are consistent with those using similar methodology in man,, 8 which suggest that the maximal pressure-volume point is more rapidly achieved in the unloaded condition compared with that in the resistively loaded condition, and may explain in part why the slope and $V_{O}$ are larger for $\mathrm{E}_{\max }$ compared with $\mathrm{E}_{e s}$ in some dogs. Further, Miller et al. ${ }^{23}$ reported a disproportionate sensitivity of the regional $\mathrm{E}_{\mathrm{es}}$ slope values to variations in arterial loading conditions in contrast to the regional $\mathrm{E}_{\max }$ slope values, which further supports the importance of the timing of systolic events on the calculation of these contractile indices, particularly $\mathrm{E}_{\mathrm{es}}$. A third explanation may be the difficulty inherent in this preparation of identifying the maximal pressure-volume ratios from each pressure-volume loop for the calculation of $\mathrm{E}_{\mathrm{es}}$ due to several pressure-volume data points being at or near the same ratio. This was not a difficulty with the $E_{\max }$ slope values, which were consistently and reproducibility identified in these animals. Consequently, the explanation for differ- 
ences in the average $E_{\max }$ and $E_{\text {es }}$ slope values may be complex and may be due to curvilinearity, the timing of systolic events, particularly in the unloaded condition, and the ability to identify the appropriate pressure-volume data points to include when calculating $\mathrm{E}_{\mathrm{es}}$ slope values.

The problem of delineating the mechanisms for the differences in the mean $\mathbf{E}_{\max }$ slope values and other contractile indices in man is that of insufficient data points to determine whether curvilinearity exists and in obtaining sufficient pressure-volume data points to guarantee statistical reliability of the slope values. A minimum of three pressure-volume data points have been employed to calculate $\mathrm{E}_{\max }$ and other contractile indices in man. ${ }^{6-8}$ Although these studies have identified differences between the average $E_{\max }$ and $E_{\text {es }}$ slope values, the statistical reliability of the individual slope values may not be sufficient to conclude that the $\mathrm{E}_{\max }$ and $\mathrm{E}_{\mathrm{es}}$ slope values in an individual patient differ significantly, or to elucidate the mechanisms for these differences, if indeed they do differ in an individual patient, because their 95\% confidence intervals are wide. In our study, when a $z$ test for parallelism of the $\mathrm{E}_{\max }$ and $\mathrm{E}_{\mathrm{es}}$ slope values was performed to determine whether these slope values differed in each dog, no significant difference was noted when up to seven pressure-volume data points were included in the calculations. These observations imply that the use of few pressure-volume data points to calculate either the $\mathrm{E}_{\max }$ or $\mathrm{E}_{\mathrm{es}}$ relationship may be insufficient to determine whether a statistically significant change in left ventricular contractility has occurred in an individual animal or patient. However, the $\mathrm{E}_{\max }$ or $\mathrm{E}_{\mathrm{es}}$ data may be used to assess differences in left ventricular contractility between patient groups or to determine whether changes in left ventricular contractility have occurred in response to an intervention within groups.

It has also been suggested on theoretic grounds that $\mathrm{E}_{\max }$ and $\mathrm{E}_{\mathrm{es}}$ slope values may have to be standardized to eliminate the influence of heart size before differences in left ventricular contractile state can be identified..$^{15}$ Belcher et al..$^{9}$ reported that $E_{\mathrm{es}}$ slope values demonstrated an inverse linear relationship with dog weight and left ventricular weight under basal and enhanced contractile states. Similarly, it has been shown in patients with normal left ventricles $^{6}$ that $\mathbf{E}_{\max }$ slope values are inversely related to left ventricular mass, which is consistent with the observations of Belcher et al., ${ }^{9}$ but they were also inversely related to left ventricular end-diastolic volume. The data in the present investigation suggest that $E_{\max }$ and $E_{\text {es }}$ slope values calculated with radionuclide angiography are inversely related to left ventricular end-diastolic volume. In contrast, no independent relationship could be defined for animal weight or left ventricular weight. Only the $E_{\max }$ volume-axis intercepts were related to body and left ventricular weight. Thus the data in the present investigation are consistent with the previous observations made in various animal preparations $\mathrm{s}^{9,10}$ and in man, ${ }^{6}$ which indicate that indices of left ventricular contractility have a relationship with heart size.

Methods for standardizing the $\mathrm{E}_{\max }$ and $\mathrm{E}_{\mathrm{es}}$ slope values have been proposed..$^{10,15,24,25}$ Berko et al. ${ }^{10}$ have demonstrated, in an acute animal preparation of mitral regurgitation, that $\mathrm{E}_{\mathrm{es}}$ slope values were inversely related to left ventricular end-diastolic volume, both in the control and mitral regurgitant states. The mean $\mathrm{E}_{\mathrm{es}}$ slope value decreased from the control condition following the production of acute mitral regurgitation. This reduction in the $\mathrm{E}_{\mathrm{es}}$ slope values was presumably due to an increase in left ventricular end-diastolic volume, since the $\mathrm{E}_{\mathrm{es}}$ slope values were unchanged following standardization for the change in heart size. These data would suggest that $E_{\max }$ and $E_{\mathrm{es}}$ slope values should be standardized for the change in heart size to determine whether a change in left ventricular contractility has occurred. In contrast, others have suggested standardizing ${ }^{15}$ or have tried standardizing ${ }^{24,25}$ contractile indices by multiplying the $\mathrm{E}_{\mathrm{es}}$ slope by the $\mathrm{V}_{0}$. However, using an extrapolated value obtained assuming linearity between pressure-volume data points may not be appropriate, because the relationship may be curvilinear and negative $V_{O}$ values may be obtained in some patients or animals. Consequently, simple multiplication of $E_{\max }$ and $E_{e s}$ slope values by $V_{O}$, considering the demonstrated problems with determining $V_{O}$ in this and other investigations, ${ }^{20,21}$ suggests that other approaches should be investigated.

Whether standardization of $E_{\max }$ slope values is necessary to determine if left ventricular contractility is normal or depressed in an individual patient, when comparisons are made between patients who have a normal left ventricle and patients who have an increase in heart size due to cardiac pathology, is not clear. ${ }^{24,26}$ Using a mathematical standardization of the $\mathrm{E}_{\max }$ slope value for left ventricular end-diastolic volume, preliminary data from our laboratory would suggest that, at least in patients with left ventricular volume overload and cardiomyopathy, the pathophysiologic process has a much greater impact on left ventricular contractility than does heart size. ${ }^{26}$ The percentage reduction in the $E_{\max }$ slope values due to heart size averaged only $13 \%$. This was similar to the reduction in the $\mathrm{E}_{\mathrm{es}}$ slope value observed by Berko et al. ${ }^{10}$ following the development of acute mitral re- 
gurgitation in an animal preparation. Consequently, a correction for heart size in patients with chronic left ventricular volume overload or cardiomyopathy did not help dichotomize patients into those with preserved compared to depressed left ventricular contractility. ${ }^{26}$ Thus standardization for heart size is probably important when one compares contractile indices generated in the same heart under different conditions, while it may not be necessary for determining whether left ventricular contractility is preserved or depressed between patients.

Potential limitations to the methodology used to calculate the maximum time-varying elastance in this short-term animal preparation should be considered. We employed aortic or inferior vena caval occlusion to alter left ventricular loading conditions. Previous studies have demonstrated that altering arterial resistance by pharmacologic means shifts the end-systolic pressure-volume relationship to a smaller $V_{0}$ without a change in slope from that observed under control conditions. ${ }^{16,27,28}$ This probably relates to a closer approximation of the isovolumic length-tension relationship when arterial load is increased. ${ }^{27}$ Nevertheless, the changes observed in these studies were small and were well within the potential variability of all clinically applicable volumetric techniques.

Second, it has also been demonstrated that the pressure-volume relationship may be curvilinear at the extremes of the pressure range. ${ }^{21,22,29,30}$ In the puppy left ventricle, Suga et al. ${ }^{30}$ demonstrated a plateauing of the end-systolic pressure-volume relationship at high arterial loads, suggesting a saturation effect. This was not observed, however, in the adult canine left ventricle. Sunagawa et al. ${ }^{29}$ have observed a curvilinear relationship toward the volume-axis intercept at loads below a critical coronary perfusion pressure of approximately $60 \mathrm{~mm} \mathrm{Hg}$. This may be related to inadequate coronary perfusion and the induction of global myocardial ischemia. We did not alter left ventricular loading conditions in these animals below a mean arterial pressure of $60 \mathrm{~mm} \mathrm{Hg}$. Moreover, curvilinearity of the end-systolic pressurevolume relationship has been observed by Burkhoff et al. ${ }^{20}$ at high or low left ventricular contractile states, while linear relationships were observed within the range of contractile states observed in some of our animals. As previously discussed, curvilinearity did exist in our short-term animal preparations for both the $\mathbf{E}_{\max }$ and $\mathrm{E}_{\mathrm{es}}$ slope values in the higher contractile range, which may explain in part the difference between the average $E_{\max }$ and $E_{e s}$ slope values. Therefore one may only assume linearity for these relationships within a relatively narrow range of contractile states. Whether curvilinearity of these relationships exists in man will require the generation of many more pressure-volume data points.

Finally, we studied our animals following betaadrenergic blockade to alleviate the effects of alterations in sympathetic nervous system activity on left ventricular contractility. ${ }^{31,32}$ We cannot completely exclude reflex changes in autonomic tone during the alterations in loading conditions produced by the aortic or inferior vena caval occlusions. Previous data from our laboratory in man would suggest, however, that this range of loading conditions produces only a modest change in serum catecholamines and no change in left ventricular contractility, as measured by $(+) \mathrm{dP} / \mathrm{dt}$ at a developed pressure of $40 \mathrm{~mm} \mathrm{Hg}$. This observation is consistent with prior observations on the effects of catecholamines on left ventricular contractility in conscious animals. ${ }^{33,34}$ Thus alterations in the activity of the sympathetic nervous system probably had little effect on the generation of the $\mathrm{E}_{\max }$ and $\mathrm{E}_{\mathrm{es}}$ slope values in our animals.

We conclude that the mean $E_{\max }$ and $E_{e s}$ slope values calculated with radionuclide angiography differ. These observations are related to the assumption of linearity when curvilinearity is present, the timing of systolic events, or to a combination of these factors. Thus since both the $E_{\max }$ and $E_{e s}$ slope values calculated using the radionuclide methodology employed in this investigation are variably affected by similar influences, are highly correlated, are not different in an individual animal, and are comparably related to heart size, they both may be useful for assessing short- and long-term alteration in left ventricular contractility in animals and man, as long as they are applied consistently within an individual animal or patient or between groups of animals or patients.

The assistance of Sheila Squicciarini, BS, and Diane Bauer in the preparation of this manuscript is appreciated.

\section{REFERENCES}

1. Suga H, Sagawa K, Shoukas AA. Load independence of the instantaneous pressure-volume ratio of the canine left ventricle and effects of epinephrine and heart rate on the ratio. Circ Res 1973;32:314.

2. Suga H, Sagawa K. Instantaneous pressure-volume relationships and their ratio in the excised, supported canine left ventricle. Circ Res 1974;35:117.

3. Sagawa K. The ventricular pressure-volume diagram revis ited. Circ Res 1978;43:677.

4. Little WC, Freeman GL. Description of $\mathrm{I} / \mathrm{V}$ pressure-volume relations by time-varying elastance and source resistance. Am J Physiol 1987;253:H83.

5. McKay RG, Aroesty JM, Heller GV, Royal HD, Warren SE, Grossman W. Assessment of the end-systolic pressure-volume relationship in human beings with the use of a time-varying elastance model. Circulation 1986;74:97.

6. Starling MR, Walsh RA, Dell'Italia LJ, Mancini GBJ, Lasher JC, Lancaster JL. The relationship of various measures of 
end-systole to left ventricular maximum time-varying elastance in man. Circulation 1987;76:32

7. Starling MR, Gross MD, Walsh RA, Dell'Italia LJ, Montgomery DG, Squicciarini SA, Blumhardt R. Assessment of the radionuclide angiographic left ventricular maximum timevarying elastance calculation in man. J Nucl Med 1988;29:1368.

8. Starling MR, Gross MD, Walsh RA, Mancini GBJ, Blumhardt R. Radionuclide determination of the relationship between left ventricular contractile state and ejection fraction. AM HEART J 1988;116:790.

9. Belcher P, Boerboom LE, Olinger GN. Standardization of end-systolic pressure-volume relation in the dog. Am J Physiol 1985;249:H547.

10. Berko B, Grasch WH, Tanigawa N, Smith D, Craige E. Disparity between ejection and end-systolic indexes of left ventricular contractility in mitral regurgitation. Circulation 1987;75:1310.

11. Starling MR, Montgomery DG, Mancini GBJ, Walsh RA. Load independence of the rate of isovolumic relaxation in man. Circulation 1987;76:1274.

12. Starling MR, Dell'Italia LJ, Walsh RA, Little WC, Benedetto AR. Accurate estimates of absolute left ventricular volumes from equilibrium radionuclide angiographic count data using a simple geometric attenuation correction. J Am Coll Cardiol 1984;3:789

13. Starling MR, Dell'Italia Lu, Nusynowitz. ML, Walsh RA, Little WC, Benedetto AR. Estimates of left-ventricular volumes by equilibrium radionuclide angiography: importance of attenuation correction. J Nucl Med 1984;25:14.

14. Mehmel HC, Stockins B, Ruffmann K, von Olshausen K Schuler G, Kubler W. The linearity of the end-systolic pressure-volume relationship in man and its sensitivity for assessment of left ventricular function. Circulation 1981;63:1216.

15. Sagawa K. The end-systolic pressure-volume relation of the ventricle: definition, modifications and clinical use. Circulation 1981;63:1223.

16. Sodums MT, Badke FR, Starling MR, Little WC, O'Rourke RA. Evaluation of left ventricular contractile performance utilizing end-systolic pressure-volume relationships in conscious dogs. Circ Res 1984;54:731.

17. Little WC, Freeman GL, O'Rourke RA. Simultaneous determination of left ventricular end-systolic pressure-volume and pressure-dimension relationships in closed-chest dogs. Circulation 1985;71:1301.

18. Kaseda S, Tomoike H, Ogata I, Nakamura M. End-systolic pressure-volume, pressure-length, and stress-strain relations in canine hearts. Am J Physiol 1985;249:H648

19. Igarashi $Y$, Suga $H$. Assessment of slope of end-systolic pressure-volume line of in situ dog heart. Am J Physiol 1986 250:H685.
20. Burkhoff D, Sugiura S, Yue DT, Sagawa K. Contractility-dependent curvilinearity of end-systolic pressure-volume relations. Am J Physiol 1987;252:H1218.

21. Little WC, Cheng C-P, Peterson T, Vinten-Johansen J. Response of left ventricular end-systolic pressure-volume relation in conscious dogs to a wide range of contractile states. Circulation 1988;78:736.

22. Kass DA, Maughan WL. From 'Emax' to pressure-volume relations: a broader view. Circulation 1988;77:1203.

23. Miller WP, Flygenring BP, Nellis SH. Effects of load alteration and coronary perfusion pressure on regional end-systolic relations. Circulation 1988;78:1299.

24. Mirsky I, Corin WJ, Murakami T, Grimm J, Hess OM, Krayenbuehl HP. Correction for preload in assessment of myocardial contractility in aortic and mitral valve disease: application of the concept of systolic myocardial stiffness. Circulation 1988;78:68.

25. Suga H, Hisano R, Goto Y, Yamada O. Normalization of endsystolic pressure-volume relation and $\mathrm{E}_{\max }$ of different-sized hearts. Jpn Circ J 1984;48:136.

26. Hsia HH, Starling MR. Is it necessary to standardize left ventricular elastance for heart size? Circulation 1988;78:523.

27. Maughan WL, Sunagawa K, Burkhoff D, Sagawa K. Effect of arterial impedance changes on the end-systolic pressurevolume relation. Circ Res 1984;54:595.

28. Freeman GL, Little WC, O'Rourke RA. The effect of vasoactive agents on the left ventricular end-systolic pressurevolume relation in closed-chest dogs. Circulation 1986;74:1107.

29. Sunagawa K, Maughan WL, Friesinger G, Gunzman $P$, Chang M-S, Sagawa K. Effects of coronary arterial pressure on left ventricular end-systolic pressure-volume relation of isolated canine heart. Circ Res 1982;50:727.

30. Suga H, Yamada O, Goto Y, Igarashi Y. Peak isovolumic pressure-volume relation of puppy left ventricle. Am J Physiol 1986;250:H167.

31. Spratt JA, Tyson GS, Glower DD, Davis JW, Mulhbaier LH, Olsen CO, Rankin JS. The end-systolic pressure-volume relationship in conscious dogs. Circulation 1987;75:1295.

32. Maughan WL, Sunagawa K, Burkhoff D, Graves WL Jr, Hunter WC, Sagawa K. Effect of heart rate on the canine endsystolic pressure-volume relationship. Circulation 1985;72:654.

33. Hintze TH, Vatner SF. Cardiac dynamics during hemorrhage: relative unimportance of adrenergic inotropic responses. Circ Res 1982;50:705.

34. Young MA, Hintze TH, Vatner SF. Correlation between cardiac performance and plasma catecholamine levels in conscious dogs. Am J Physiol 1985;248:H82. 